\title{
Physiologic Monitor Alarm Rates at 5 Children's Hospitals
}

\author{
Amanda C. Schondelmeyer, MD, MSc ${ }^{1,2, *}$, Patrick W. Brady, MD, MSc ${ }^{1,2}$, Veena V. Goel, MD ${ }^{3,4}$, Maria Cvach, DNP, RN, \\ Nancy Blake, PhD, RN7, Colleen Mangeot, $\mathrm{MS}^{8}$, Christopher P. Bonafide, MD, MSCE
}

\begin{abstract}
'Division of Hospital Medicine, Department of Pediatrics, Cincinnati Children's Hospital Medical Center, Cincinnati, Ohio; ${ }^{2} J a m e s ~ M . ~ A n d e r s o n$ Center for Health Systems Improvement, Cincinnati Children's Hospital Medical Center, Cincinnati, Ohio; ${ }^{3}$ Division of Pediatric Hospital Medicine, Palo Alto Medical Foundation, Sutter Health, Palo Alto, California; ${ }^{4}$ Division of Hospital Medicine, Lucile Packard Children's Hospital Stanford, Palo Alto, California; ${ }^{5}$ Department of Nursing, Johns Hopkins Hospital, Baltimore, Maryland; 'Department of Integrated Healthcare Delivery, Johns Hopkins Health System, Baltimore, Maryland; 'Division of Nursing, Children's Hospital Los Angeles, California; ${ }^{8}$ Division of Biostatistics and Epidemiology, Cincinnati Children's Hospital Medical Center, Cincinnati, Ohio; ${ }^{9}$ Division of General Pediatrics, Children's Hospital of Philadelphia, Philadelphia, Pennsylvania.
\end{abstract}

\begin{abstract}
Alarm fatigue has been linked to patient morbidity and mortality in hospitals due to delayed or absent responses to monitor alarms. We sought to describe alarm rates at 5 freestanding children's hospitals during a single day and the types of alarms and proportions of patients monitored by using a point-prevalence, cross-sectional study design. We collected audible alarms on all inpatient units and calculated overall alarm rates and rates by alarm type per monitored patient per day. We found a total of 147,213 alarms during
\end{abstract}

the study period, with 3-fold variation in alarm rates across hospitals among similar unit types. Across hospitals, onequarter of monitored beds were responsible for $71 \%, 61 \%$, and $63 \%$ of alarms in medical-surgical, neonatal intensive care, and pediatric intensive care units, respectively. Future work focused on addressing nonactionable alarms in patients with the highest alarm counts may decrease alarm rates. Journal of Hospital Medicine 2018;13:396-398. Published online first April 25, 2018. (c) 2018 Society of Hospital Medicine

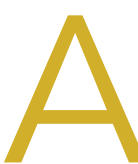

larm fatigue is a patient safety hazard in hospitals that occurs when exposure to high rates of alarms leads clinicians to ignore or delay their responses to the alarms. ${ }^{2,3}$ To date, most studies of physiologic monitor alarms in hospitalized children have used data from single institutions and often only a few units within each institution. ${ }^{4}$ These limited studies have found that alarms in pediatric units are rarely actionable. ${ }^{2}$ They have also shown that physiologic monitor alarms occur frequently in children's hospitals and that alarm rates can vary widely within a single institution, ${ }^{5}$ but the extent of variation between children's hospitals is unknown. In this study, we aimed to describe and compare physiologic monitor alarm characteristics and the proportion of patients monitored in the inpatient units of 5 children's hospitals.

\section{METHODS}

We performed a cross-sectional study using a point-prevalence design of physiologic monitor alarms and monitoring during a 24-hour period at 5 large, freestanding tertiary-care

\footnotetext{
*Address for correspondence: Amanda C. Schondelmeyer, MD, MSc, Cincinnati Children's Hospital Medical Centre, 3333 Burnet Ave ML 9016, Cincinnati, $\mathrm{OH}$ 45229; Telephone: 513-803-9158; Fax: 513-803-9244; E-mail: amanda. schondelmeyer@cchmc.org

Additional Supporting Information may be found in the online version of this article.
}

Received: August 3, 2017; Revised: October 6, 2017;

Accepted: October 19, 2017

(C) 2018 Society of Hospital Medicine DOI 10.12788/jhm.2918 children's hospitals. At the time of the study, each hospital had an alarm management committee in place and was working to address alarm fatigue. Each hospital's institutional review board reviewed and approved the study.

We collected 24 consecutive hours of data from the inpatient units of each hospital between March 24, 2015, and May 1, 2015. Each hospital selected the data collection date within that window based on the availability of staff to perform data collection. ${ }^{6}$ We excluded emergency departments, procedural areas, and inpatient psychiatry and rehabilitation units. By using existing central alarm-collection software that interfaced with bedside physiologic monitors, we collected data on audible alarms generated for apnea, arrhythmia, low and high oxygen saturation, heart rate, respiratory rate, blood pressure, and exhaled carbon dioxide. Bedside alarm systems and alarm collection software differed between centers; therefore, alarm types that were not consistently collected at every institution (eg, alarms for electrode and device malfunction, ventilators, intracranial and central venous pressure monitors, and temperatures probes) were excluded. To estimate alarm rates and to account for fluctuations in hospital census throughout the day, $^{7}$ we collected census (to calculate the number of alarms per patient day) and the number of monitored patients (to calculate the number of alarms per monitored-patient day, including only monitored patients in the denominator) on each unit at 3 time points, 8 hours apart. Patients were considered continuously monitored if they had presence of a waveform and data for pulse oximetry, respiratory rate, and/or heart rate at the time of data collection. We then determined the rate of alarms by unit type - medical-surgical unit (MSU), neonatal in- 
TABLE 1. Median Alarm Rate Per Patient Day and Per Monitored-Patient Day and Percentage of Patients Monitored by Hospital and Unit Type

\begin{tabular}{|c|c|c|c|c|c|c|}
\hline & & \multicolumn{5}{|c|}{ Hospital } \\
\hline & & A & B & C & D & $\mathrm{E}$ \\
\hline \multirow[t]{3}{*}{ MSU } & Percentage Monitored & $32 \%$ & $48 \%$ & $38 \%$ & $40 \%$ & $26 \%$ \\
\hline & Alarms per patient day & 15 & 20 & 34 & 45 & 40 \\
\hline & Alarms per monitored-patient day & 42 & 50 & 78 & 104 & 155 \\
\hline \multirow[t]{3}{*}{$\mathrm{NICU}$} & Percentage Monitored & $100 \%$ & $97 \%$ & $100 \%$ & $100 \%$ & $100 \%$ \\
\hline & Alarms per patient day & 133 & 135 & 153 & 115 & 351 \\
\hline & Alarms per monitored-patient day & 133 & 140 & 153 & 115 & 351 \\
\hline \multirow[t]{3}{*}{ PICU } & Percentage Monitored & $100 \%$ & $100 \%$ & $100 \%$ & $100 \%$ & $100 \%$ \\
\hline & Alarms per patient day & 104 & 54 & 235 & 112 & 310 \\
\hline & Alarms per monitored-patient day & 104 & 54 & 235 & 112 & 310 \\
\hline
\end{tabular}

NOTE: Abbreviations: MSU, medical-surgical unit; NICU, neonatal intensive care unit; PICU, pediatric intensive care unit.

tensive care unit (NICU), or pediatric intensive care unit (PICU) - and the alarm types. Based on prior literature demonstrating up to $95 \%$ of alarms contributed by a minority of patients on a single unit, ${ }^{8}$ we also calculated the percentage of alarms contributed by beds in the highest quartile of alarms. We also assessed the percentage of patients monitored by unit type. The Supplementary Appendix shows the alarm parameter thresholds in use at the time of the study.

\section{RESULTS}

A total of 147,213 eligible clinical alarms occurred during the 24-hour data collection periods in the 5 hospitals. Alarm rates differed across the 5 hospitals, with the highest alarm hospitals having up to 3-fold higher alarm rates than the lowest alarm hospitals (Table 1). Rates also varied by unit type within and across hospitals (Table 1). The highest alarm rates overall during the study occurred in the NICUs, with a range of 115 to 351 alarms per monitored patient per day, followed by the PICUs (range 54-310) and MSUs (range 42-155).

While patient monitoring in the NICUs and PICUs was nearly universal (97\%-100\%) at institutions during the study period, a range of $26 \%$ to $48 \%$ of beds were continuously monitored in MSUs. Of the 12 alarm parameters assessed, low oxygen saturation had the highest percentage of total alarms in both the MSUs and NICUs for all hospitals, whereas the alarm parameter with the highest percentage of total alarms in the PICUs varied by hospital. The most common alarm types in 2 of the 5 PICUs were high blood pressure alarms and low pulse oximetry, but otherwise, this varied across the remainder of the units (Table 2).

Averaged across study hospitals, one-quarter of the monitored beds were responsible for $71 \%$ of alarms in MSUs, $61 \%$ of alarms in NICUs, and $63 \%$ of alarms in PICUs.
TABLE 2. Top 3 Alarm Parameters with the Highest Percentage of Total Alarms by Hospital and Unit

\begin{tabular}{|c|c|c|c|}
\hline Hospital & MSU & $\mathrm{NICU}$ & PICU \\
\hline \multirow[t]{3}{*}{$A$} & SPO2 low (41\%) & SPO2 low (61\%) & BP high (25\%) \\
\hline & HR low (35\%) & SPO2 high (19\%) & BP low (21\%) \\
\hline & HR high (21\%) & HR low (11\%) & SPO2 low (15\%) \\
\hline \multirow[t]{3}{*}{$B$} & SPO2 low (44\%) & SPO2 low (30\%) & SPO2 low (31\%) \\
\hline & HR high (16\%) & SPO2 high (25\%) & RR low (20\%) \\
\hline & RR high (11\%) & RR low (15\%) & HR high (15\%) \\
\hline \multirow[t]{3}{*}{$C$} & SPO2 low (36\%) & SPO2 low (45\%) & BP high (24\%) \\
\hline & RR high (21\%) & HR low (36\%) & SPO2 low (20\%) \\
\hline & RR low (14\%) & HR high (10\%) & BP low (17\%) \\
\hline \multirow[t]{3}{*}{$D$} & SPO2 low (24\%) & SPO2 low (44\%) & Arrhythmia (31\%) \\
\hline & HR high (19\%) & HR low (14\%) & SPO2 low (18\%) \\
\hline & RR high (17\%) & RR low (14\%) & HR high (13\%) \\
\hline \multirow[t]{3}{*}{$E$} & SPO2 low (38\%) & SPO2 low (48\%) & SPO2 low (26\%) \\
\hline & RR high (15\%) & RR high (28\%) & Arrhythmia (20\%) \\
\hline & HR high (15\%) & RR low $(9 \%)$ & HR high (15\%) \\
\hline
\end{tabular}

NOTE: Abbreviations: $\mathrm{HR}$, heart rate; MSU, medical-surgical unit; NICU, neonatal intensive care unit; PICU, pediatric intensive care unit; RR, respiratory rate; SPO2, oxygen saturation.

\section{DISCUSSION}

Physiologic monitor alarm rates and the proportion of patients monitored varied widely between unit types and among the tertiary-care children's hospitals in our study. We found that among MSUs, the hospital with the lowest proportion of beds monitored had the highest alarm rate, with over triple the rate seen at the hospital with the lowest alarm rate. Regardless of unit type, a small subgroup of patients at each hospital contributed a disproportionate share of alarms. These findings are concerning because of the patient morbidity and mortality associated with alarm fatigue ${ }^{1}$ and the studies suggesting that higher alarm rates may lead to delays in response to potentially critical alarms. ${ }^{2}$

We previously described alarm rates at a single children's hospital and found that alarm rates were high both in and outside of the ICU areas. ${ }^{5}$ This study supports those findings and goes further to show that alarm rates on some MSUs approached rates seen in the ICU areas at other centers. ${ }^{4}$ However, our results should be considered in the context of several limitations. First, the 5 study hospitals utilized different bedside monitors, equipment, and software to collect alarm data. It is possible that this impacted how alarms were counted, though there were no technical specifications to suggest that results should have been biased in a specific way. Second, our data did not reflect alarm validity (ie, whether an alarm accurately reflected the physiologic state of the patient) or factors outside of the number of 
patients monitored - such as practices around ICU admission and transfer as well as monitor practices such as lead changes, the type of leads employed, and the degree to which alarm parameter thresholds could be customized, which may have also affected alarm rates. Finally, we excluded alarm types that were not consistently collected at all hospitals. We were also unable to capture alarms from other alarm-generating devices, including ventilators and infusion pumps, which have also been identified as sources of alarm-related safety issues in hospitals. ${ }^{9-11}$ This suggests that the alarm rates reported here underestimate the total number of audible alarms experienced by staff and by hospitalized patients and families.

While our data collection was limited in scope, the striking differences in alarm rates between hospitals and between similar units in the same hospitals suggest that unit- and hospital-level factors-including default alarm parameter threshold settings, types of monitors used, and monitoring practices such as the degree to which alarm parameters are customized to the patient's physiologic state-likely contribute to the variability. It is also important to note that while there were clear outlier hospitals, no single hospital had the lowest alarm rate across all unit types. And while we found that a small number of patients contributed disproportionately to alarms, monitoring fewer patients overall was not consistently associated with lower alarm rates. While it is difficult to draw conclusions based on a limited study, these findings suggest that solutions to meaningfully lower alarm rates may be multifaceted. Standardization of care in multiple areas of medicine has shown the potential to decrease unnecessary utilization of testing and therapies while maintaining good patient outcomes. ${ }^{12-15}$ Our findings suggest that the concept of positive deviance, ${ }^{16}$ by which some organizations produce better outcomes than others despite similar limitations, may help identify successful alarm reduction strategies for further testing. Larger quantitative studies of alarm rates and ethnographic or qualitative studies of monitoring practices may reveal practices and policies that are associated with lower alarm rates with similar or improved monitoring outcomes.

\section{CONCLUSION}

We found wide variability in physiologic monitor alarm rates and the proportion of patients monitored across 5 children's hospitals. Because alarm fatigue remains a pressing patient safety concern, further study of the features of high-performing (low-alarm) hospital systems may help identify barriers and facilitators of safe, effective monitoring and develop targeted interventions to reduce alarms.

\section{Acknowledgments}

The authors thank Melinda Egan, Matt MacMurchy, and Shannon Stemler for their assistance with data collection.

Disclosure: Dr. Bonafide is supported by the National Heart, Lung, and Blood Institute of the National Institutes of Health under Award Number K23HL116427. Dr. Brady is supported by the Agency for Healthcare Research and Quality under Award Number K08HS23827. The content is solely the responsibility of the authors and does not necessarily represent the official views of the National Institutes of Health or the Agency for Healthcare Research and Quality. There was no external funding obtained for this study. The authors have no conflicts of interest to disclose.

\section{References}

1. Sentinel Event Alert Issue 50: Medical device alarm safety in hospitals. The Joint Commission. April 8, 2013. www.jointcommission.org/sea_issue_50. Accessed December 16, 2017.

2. Bonafide $C P$, Lin $R$, Zander $M$, et al. Association between exposure to nonactionable physiologic monitor alarms and response time in a children's hospital. J Hosp Med. 2015;10(6):345-351.

3. Voepel-Lewis $\mathrm{T}$, Parker $\mathrm{ML}$, Burke $\mathrm{CN}$, et al. Pulse oximetry desaturation alarms on a general postoperative adult unit: A prospective observational study of nurse response time. Int J Nurs Stud. 2013;50(10):1351-1358.

4. Paine CW, Goel W, Ely E, et al. Systematic review of physiologic monitor alarm characteristics and pragmatic interventions to reduce alarm frequency. J Hosp Med. 2016;11(2):136-144.

5. Schondelmeyer AC, Bonafide CP, Goel W, et al. The frequency of physiologic monitor alarms in a children's hospital. J Hosp Med. 2016;11(11):796-798.

6. Zingg W, Hopkins S, Gayet-Ageron A, et al. Health-care-associated infections in neonates, children, and adolescents: An analysis of paediatric data from the European Centre for Disease Prevention and Control point-prevalence survey. Lancet Infect Dis. 2017;17(4):381-389.

7. Fieldston E, Ragavan M, Jayaraman B, Metlay J, Pati S. Traditional measures of hospital utilization may not accurately reflect dynamic patient demand: Findings from a children's hospital. Hosp Pediatr. 2012;2(1):10-18.

8. Cvach M, Kitchens M, Smith K, Harris P, Flack MN. Customizing alarm limits based on specific needs of patients. Biomed Instrum Technol. 2017;51(3):227234

9. Pham JC, Williams TL, Sparnon EM, Cillie TK, Scharen HF, Marella WM. Ventilator-related adverse events: A taxonomy and findings from 3 incident reporting systems. Respir Care. 2016;61(5):621-631.

10. Cho OM, Kim H, Lee YW, Cho I. Clinical alarms in intensive care units: Perceived obstacles of alarm management and alarm fatigue in nurses. Healthc Inform Res. 2016;22(1):46-53.

11. Edworthy J, Hellier E. Alarms and human behaviour: Implications for medical alarms. Br J Anaesth. 2006;97(1):12-17.

12. Fisher ES, Wennberg DE, Stukel TA, Gottlieb DJ, Lucas FL, Pinder EL. The implications of regional variations in medicare spending. Part 1: The content, quality, and accessibility of care. Ann Intern Med. 2003;138(4):273-287.

13. Fisher ES, Wennberg DE, Stukel TA, Gottlieb DJ, Lucas FL, Pinder EL. The implications of regional variations in medicare spending. Part 2: Health outcomes and satisfaction with care. Ann Intern Med. 2003;138(4):288-298

14. Lion KC, Wright DR, Spencer S, Zhou C, Del Beccaro M, Mangione-Smith R. Standardized clinical pathways for hospitalized children and outcomes. Pediatrics. 2016;137(4) e20151202.

15. Goodman DC. Unwarranted variation in pediatric medical care. Pediatr Clin North Am. 2009;56(4):745-755

16. Baxter R, Taylor N, Kellar I, Lawton R. What methods are used to apply positive deviance within healthcare organisations? A systematic review. BMJ Qual Saf. 2016;25(3):190-201 